\title{
Chronic traumatic encephalopathy is a common co-morbidity, but less frequent primary dementia in former soccer and rugby players
}

\author{
Edward B. Lee ${ }^{1} \cdot$ Kevin Kinch ${ }^{2} \cdot$ Victoria E. Johnson ${ }^{3} \cdot$ John Q. Trojanowski ${ }^{4} \cdot$ Douglas H. Smith $^{3}$. \\ William Stewart ${ }^{2,5}$
}

Received: 14 May 2019 / Accepted: 25 May 2019 / Published online: 1 June 2019

(c) The Author(s) 2019

\begin{abstract}
Chronic traumatic encephalopathy (CTE) is reported at high prevalence in selected autopsy case series of former contact sports athletes. Nevertheless, the contribution of CTE pathology to clinical presentation and its interaction with co-morbid neurodegenerative pathologies remain unclear. To address these issues, we performed comprehensive neuropathology assessments on the brains of former athletes with dementia and considered these findings together with detailed clinical histories to derive an integrated clinicopathological diagnosis for each case. Consecutive, autopsy-acquired brains from former soccer and rugby players with dementia were assessed for neurodegenerative pathologies using established and preliminary consensus protocols. Thereafter, next of kin interviews were conducted to obtain detailed accounts of the patient's clinical presentation and course of disease to inform a final, integrated clinicopathological diagnosis. Neuropathologic change consistent with CTE (CTE-NC) was confirmed in five of seven former soccer and three of four former rugby players' brains, invariably in combination with mixed, often multiple neurodegenerative pathologies. However, in just three cases was the integrated dementia diagnosis consistent with CTE, the remainder having alternate diagnoses, with the most frequent integrated diagnosis Alzheimer's disease (AD) (four cases; one as mixed $\mathrm{AD}$ and vascular dementia). This consecutive autopsy series identifies neuropathologic change consistent with preliminary diagnostic criteria for CTE (CTE-NC) in a high proportion of former soccer and rugby players dying with dementia. However, in the majority, CTE-NC appears as a co-morbidity rather than the primary, dementia causing pathology. As such, we suggest that while CTE-NC might be common in former athletes with dementia, in many cases its clinical significance remains uncertain.
\end{abstract}

Keywords Chronic traumatic encephalopathy $\cdot$ Traumatic brain injury $\cdot$ Neurodegeneration $\cdot$ Dementia $\cdot$ Alzheimer's disease · Tau

William Stewart

william.stewart@glasgow.ac.uk

1 Translational Neuropathology Research Laboratory, University of Pennsylvania, Philadelphia, PA 19104, USA

2 Department of Neuropathology, Queen Elizabeth University Hospital, 1345 Govan Rd, Glasgow G51 4TF, UK

3 Department of Neurosurgery, Penn Center for Brain Injury and Repair, Perelman School of Medicine, University of Pennsylvania, Philadelphia, PA 19104, USA

4 Department of Pathology and Laboratory Medicine, Center for Neurodegenerative Disease Research, Institute on Aging, Perelman School of Medicine, University of Pennsylvania, Philadelphia, USA

5 Institute of Neuroscience and Psychology, University of Glasgow, Glasgow G12 8QQ, UK

\section{Introduction}

There is growing concern over the association between exposure to traumatic brain injury (TBI) and increased risk of a variety of neuropsychiatric and neurocognitive outcomes, in particular those linked to a specific neurodegenerative pathology known as chronic traumatic encephalopathy (CTE) $[11,29,31,42,50]$. First recognized in the early part of the twentieth century as the punch drunk syndrome of boxers [24], it was not until descriptions of CTE in autopsy studies of non-boxer athletes that the lifelong consequences of exposure to TBI attracted widespread attention $[27,36]$. Nevertheless, despite this increased attention, there remain comparatively few accounts of the neuropathology of late survival from TBI [43]. As such, reflecting the relative paucity of cases described, consensus criteria for the 
neuropathological assessment of CTE remain preliminary [26] and the associated clinical consequences of this pathology remain unclear $[48,50]$.

In his report on the punch drunk syndrome, Harrison Martland provided the first, formal account of the chronic motor and neuropsychiatric consequences of exposure to repetitive mild TBI [24]. Thereafter, autopsy studies on single cases and small case series described the neuropathology of dementia pugilistica (DP) as an apparently distinctive, perivascular deposition of hyperphosphorylated tau, in the form of neurofibrillary tangles $[3,4,11,12]$. In addition, widespread amyloid- $\beta$ (A $\beta$ ) plaques were reported in a majority $[11,40]$. However, although isolated studies in nonboxer individuals exposed to repetitive TBI were reported $[6,12]$, to the end of the twentieth century accounts of DP largely were restricted to observations in former boxers.

More recently, the pathology of DP, now termed CTE, has been documented in growing numbers of non-boxer athletes; the overwhelming majority from autopsy studies in former American footballers [28, 31], in addition to isolated descriptions of former athletes from a wide and growing range of sports $[1,9,10,22,25,37,47]$, former military personnel $[8,38]$ and individuals exposed to a single moderate or severe TBI $[15,51]$. Notably, many of these contemporary studies often describe more limited and localized pathology than historical case series of DP, with perhaps more attention on neuropsychiatric than cognitive symptomatology $[42,46]$. Further, recent reporting largely focuses on tau pathologies in CTE over the constellation of non-tau proteinopathies that develop in late survivors from TBI. Reflecting this, preliminary consensus criteria for the neuropathological identification of CTE define the disease solely on the pattern and distribution of tau [26]. Finally, many reports often do not consider the clinical consequences of CTE pathology, particularly in patients with dementia where multiple neurodegenerative pathologies may co-exist.

There is, therefore, a continuing need to understand the complex neuropathology of late survival from exposure to TBI and its interaction with wider neurodegenerative pathologies. Herein, we report experience from a single institution on the neuropathological assessment of the largest series to date of former soccer (Association football) and rugby union (hereafter 'rugby') players with histories of dementia. Specifically, applying established and preliminary consensus criteria for neuropathological assessment of a range of neurodegenerative diseases, together with detailed review of the clinical histories, we provide insight into the integrated clinicopathological diagnoses of dementia in a cohort of former athletes exposed to repetitive head impacts and mild TBI. Our observations suggest that CTE neuropathologic change
(CTE-NC) is present in a high proportion of former soccer and rugby players with dementia. However, considered together, the clinical features and neuropathologies support an integrated clinicopathologic diagnosis of CTE dementia (CTE-D) in only a small proportion of these cases.

\section{Methods}

\section{Ethical approval and recruitment}

Ethical approval for all procedures in this study was provided by: the University of Glasgow Medical Veterinary and Life Sciences College Ethics Committee (reference number 200160147); the West of Scotland Research Ethics Committee (project ID 225271); and the Greater Glasgow and Clyde Biorepository (Application number 340). In each case, written authorization for a hospital diagnostic autopsy and donation of brain tissue to the Glasgow TBI Archive for use in research was obtained from the next of kin. Nevertheless, to preserve patient confidentiality, specific details of individual cases are presented as summarized data, with age at disease onset and death presented in decades. Consecutive research brain donations spanning the period 2014-2018 were included: one case each from years 2014, 2015 and 2016; two from 2017; and six from 2018. No potential donors notified to the Archive over the period of accrual were excluded.

\section{Neuropathological evaluation}

At the time of autopsy, whole brains were immersion fixed in 10\% formal saline for a minimum of 2 weeks, following which intact brains, where available, were transported to Glasgow for dissection and processing. For Case 1, only tissue blocks from target areas were available for review. Case 7 had been partially dissected prior to transport, but the complete specimen remained available for assessment. Following fixation, all brains were dissected, examined macroscopically and processed to paraffin using standard laboratory techniques. Tissue blocks for histopathological evaluation were sampled in line with standardized protocols for assessment of neurodegenerative disease. Specifically, sampled tissue blocks were sufficient to fulfill the minimum sampling recommended by the preliminary consensus criteria for CTE [26].

From all tissue blocks, $8 \mu \mathrm{m}$ sections were prepared and stained for Hematoxylin and eosin using standard laboratory techniques. In addition, in line with consensus protocols, sections from selected blocks were stained for: hyperphosphorylated tau (PHF-1; 1:1000; Dr P Davis), A $\beta$ (6f3d; 1:75; 
Dako), phosphorylated TDP-43 (1D3; 1:500; Millipore), alpha-synuclein (KM51; 1:200; Leica) and, where indicated, 3-repeat (8E6/C11; 1:3000; Millipore) and 4-repeat tau (1E1/A6; 1:400; Millipore). Thereafter, stained sections were independently reviewed by two neuropathologists (KK, WS), blind to clinical history, and a consensus reached on the nature, extent and distribution of pathologies using established criteria for neuropathological assessment of neurodegenerative disease. Specifically, preliminary NINDS consensus diagnostic criteria were employed for assessment of CTE pathology [26], with CTE neuropathologic change (CTE-NC) confirmed where hyperphosphorylated tau was present in neurons, astrocytes and cell processes around small vessels in an irregular pattern and at the depths of cortical sulci. Further, although consensus staging criteria for CTE do not exist, where present, extent and distribution of CTE-NC were dichotomized as 'low' or 'high', corresponding to stages I/II or III/IV, respectively, of a widely used protocol [28].

\section{Verbal autopsy}

Following completion of the neuropathology evaluation, face-to-face or telephone discussions (where face-to-face meeting was not practical) between the lead investigator (WS) and the next of kin were arranged. These comprised open ended, semi-structured interviews documenting a narrative account of the patient's clinical course, dementia diagnosis in life and management in the final illness. In addition, details were gathered on lifelong health (including exposure to TBI), history of participation in sport (including combat sports), medications, drug and alcohol exposure, family history and history of employment. At the culmination of this verbal autopsy, the comprehensive clinical information obtained was considered in context of the neuropathological features and a layered, diagnostic autopsy opinion provided, encompassing the final integrated diagnosis of dementia (clinicopathological diagnosis of dementia subtype) [21].

\section{Statistics}

Summary statistics and between-group differences were calculated using Minitab (Version 18.1, Minitab Inc.), with between-group differences assessed using the two-sample $t-$, Mann-Whitney or Kruskal-Wallis tests as appropriate.

\section{Results}

\section{Demographic and background information}

Eleven brain donations from former athletes with a history of dementia were received over the period of study (seven soccer; four rugby). Average age at onset of dementia was $60 \pm 8.6$ years (range 48-75), with no difference in age of onset between former soccer $(59.7 \pm 8.1$ years) or rugby $(60.5 \pm 10.8$ years; $p=0.906)$ players, and similar disease durations (soccer $9.4 \pm 4.5$ years; rugby $11.2 \pm 6.8$ years; $p=0.657$ ). Mixed playing positions were reported, with years of active participation in their sport estimated at $23.8 \pm 8.4$ years (range $11-35$ years; rugby $28.8 \pm 6.5$ years vs soccer $21 \pm 8.5$ years; $p=0.156$ ). In five subjects, a

Table 1 Cohort demographic information

\begin{tabular}{|c|c|c|c|c|c|c|c|c|}
\hline Case no & Sex & Sport & Position & $\begin{array}{l}\text { Other con- } \\
\text { tact sports }\end{array}$ & $\begin{array}{l}\text { Estimated } \\
\text { years in sport }\end{array}$ & $\begin{array}{l}\text { History of TBI } \\
\text { with LOC }\end{array}$ & Family history NDD & PMI (days) \\
\hline 1 & M & Soccer & Forward & No & 18 & NA & Nil & NA \\
\hline 2 & M & Soccer & Defense & No & 20 & Yes & Nil & 11 \\
\hline 3 & M & Soccer & Defense & No & 35 & Yes & Nil & 4 \\
\hline 4 & M & Soccer & Defense & No & 30 & No & Mother and brother 'dementia' & 3 \\
\hline 5 & M & Soccer & Forward & No & 18 & No & Nil & 1 \\
\hline 6 & M & Soccer & Defense & Rugby & 15 & No & Nil & 3 \\
\hline 7 & M & Soccer & Defense & No & 11 & No & Mother PD & 3 \\
\hline 8 & M & Rugby & Back & No & 20 & Yes & Nil & 0.5 \\
\hline 9 & M & Rugby & Forward & No & 35 & Yes & Brother AD & 2 \\
\hline 10 & M & Rugby & Forward & No & 32 & Yes & Nil & 2 \\
\hline 11 & M & Rugby & Forward & Soccer & 28 & No & Nil & 6 \\
\hline
\end{tabular}

$A D$ Alzheimer's disease, $L O C$ loss of consciousness for less than $30 \mathrm{~min}, N D D$ neurodegenerative disease, $P D$ Parkinson's disease, $P M I$ postmortem interval, TBI traumatic brain injury 

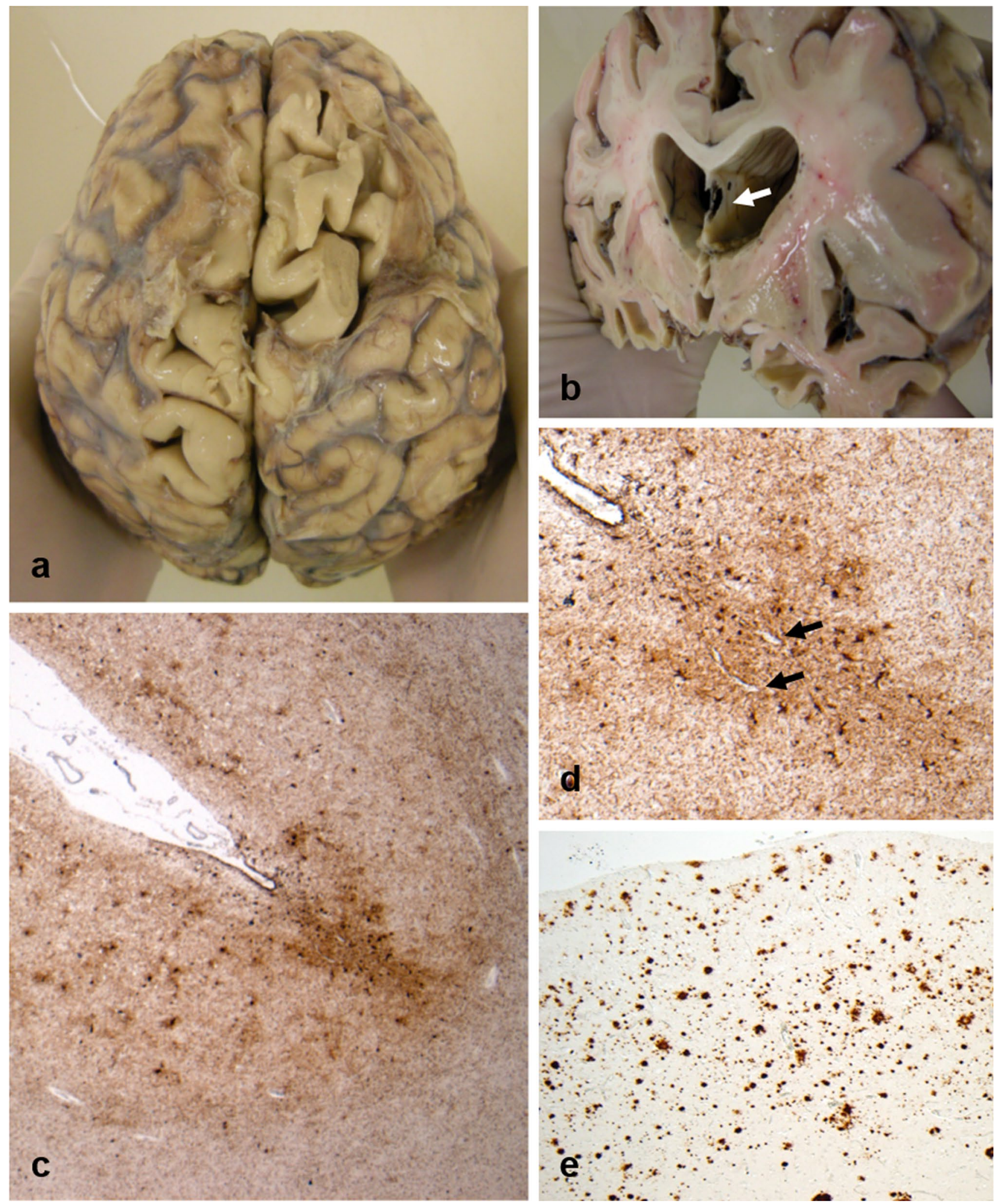
४Fig. 1 Representative macroscopic and histologic images from Case 8 , a former rugby union player who first presented in his $50 \mathrm{~s}$ with a change in behavior marked by increasing levels of frustration and agitation associated with aggressive outbursts. In the following years, these behavioral and personality symptoms progressed, with cognitive disturbance evolving late in the course of disease. The patient died in his $70 \mathrm{~s}, 18$ years after first presentation. On examination of the whole brain, there is gyral atrophy with associated sulcal thinning, most evident in frontal lobes (a). Sectioning the brain in the coronal plain through the mammillary bodies reveals ventriculomegally, consistent with the atrophy noted externally, in addition to fenestration of the septum pellucidum (b; white arrow). Staining sections for hyperphosphorylated tau (p-tau; PHF1) reveal widespread cortical immunoreactivity, within which are multiple patches of increased staining density towards the depths of cortical sulci (c), which on higher power are seen to coincide with neuronal and astroglial immunoreactive profiles clustered around small blood vessels (d; black arrows). In addition to widespread p-tau pathology, numerous $\mathrm{A} \beta$ plaques are present $(\mathbf{e} ; 6 \mathrm{f} 3 \mathrm{~d})$. The final integrated dementia diagnosis was CTE-D

history of previous TBI with brief loss of consciousness was reported; none required hospital admission and all would be compatible with 'mild' TBI/concussion. Full demographic information, including details of sports career and postmortem intervals, is provided in Table 1.

\section{Neuropathology}

\section{Macroscopic appearances}

Neuropathological evaluation revealed intact, fixed brain weights typically low (soccer $1225 \pm 118 \mathrm{~g}$; rugby $1224 \pm 120 \mathrm{~g} ; p=0.989$ ), with associated atrophy of frontal and temporal lobes (Fig. 1a) and hydrocephalus ex vacuo. In addition, moderate atrophy of the heads of the caudate nuclei was noted in Case 11. Septum pellucidum abnormalities were present in five cases, as fenestrated (Fig. 1b) or cavum septum or as mixed fenestrated and cavum (Table 2). Evidence of old, focal ischemic pathology as cavitated infarcts was noted in four cases. However, no focal pathology consistent with previous traumatic brain injury was present; specifically, no healed contusions were identified.

\section{Microscopic appearances}

Histological examination revealed a variety of neuropathologies, including those fulfilling criteria for multiple neurodegenerative pathologies (Table 2). Evidence of old ischemic injuries was confirmed in Cases 2, 4, 5, 9 and 10 as variably sized foci of architectural disturbance with neuronal loss and astrocytic gliosis, occasionally with cystic degeneration and an established glial limitans. Bilateral hippocampal sclerosis was also present in Case 2. Marked substantia nigra pigment incontinence, neuronal loss and astrocytic gliosis were noted in Cases 3, 7 and 11, and occasional remaining neurons containing either classical Lewy bodies (Case 3) or globose tangles (Cases 7 and 11).

\section{Hyperphosphorylated tau}

All 11 brains showed abnormal hyperphosphorylated tau (p-tau) pathology, consistent with several distinct tauopathies [17]. In eight cases (five soccer, three rugby), p-tau was present in a patchy distribution towards the depths of cortical sulci as neurofibrillary tangles, neurites and thornshaped astrocytes clustered around cortical vessels, fulfilling the preliminary consensus neuropathological criteria for the recognition of CTE (Figs. 1c, d, 2a, b) [26]. Notably, patients in whom CTE neuropathological change (CTE-NC) was present were generally older at disease onset (median 63.5 years vs 50 years; $p=0.019$; Mann-Whitney) and at death (74.5 years vs 59 years; $p=0.041)$ than patients without CTE-NC. There was no difference in disease duration between cases with or without CTE-NC (10.5 years vs 8.7 years; $p=0.610)$.

In addition to CTE-NC, neurofibrillary tangle pathology in wider distribution was present in six former soccer and three former rugby players, consistent with AD Braak stage III to VI pathology [2]. In the remaining two cases, p-tau pathologies appeared as 4R tau-positive, 3R tau-negative, neuronal cytoplasmic inclusions, neurites, coiled bodies, and tufted astrocytes, or as astrocytic plaques, ballooned cells, and pre-tangles, consistent with pathologic diagnoses of progressive supranuclear palsy (PSP) [23] or corticobasal degeneration (CBD), respectively [5]. Finally, subcortical, periventricular, subpial or peri-infarct p-tau immunoreactive, and thorn-shaped astrocytes were present in five cases, typical of aging-related tau astrogliopathy (ARTAG) [19, $20]$.

\section{Amyloid- $\beta$}

Screening for amyloid- $\beta$ (A $\beta$ ) pathologies revealed abnormalities in eight cases (five soccer, three rugby). In seven examples, these appeared as moderate to frequent, mixed diffuse and neuritic plaques (CERAD B or C) [32], typically in wide distribution (Thal phase 4 or 5) (Figs. 1e, 2c) 
Table 2 Neuropathological observations

\begin{tabular}{|c|c|c|c|c|c|c|c|c|c|c|c|}
\hline \multirow[t]{2}{*}{ Case no } & \multirow[t]{2}{*}{ Sport } & \multirow[t]{2}{*}{ Septum } & \multirow[t]{2}{*}{ CTE-NC } & \multicolumn{3}{|c|}{ ADNC } & \multirow[t]{2}{*}{ CAA } & \multirow[t]{2}{*}{ TDP-43 } & \multirow[t]{2}{*}{ Other ND pathology } & \multirow[t]{2}{*}{ Clinical diagnosis } & \multirow[t]{2}{*}{ Integrated CPC diagnosis } \\
\hline & & & & $A$ & $B$ & $C$ & & & & & \\
\hline 1 & Soccer & $\mathrm{F}+\mathrm{C}$ & High & 1 & 2 & 0 & 4 & - & CVD & $\mathrm{AD}$ & CTE \\
\hline 2 & Soccer & $\mathrm{C}$ & High & 0 & 2 & 0 & 0 & 5 & CVD; PART & $\mathrm{AD}$ & $\mathrm{VaD}$ \\
\hline 3 & Soccer & $\mathrm{F}$ & High & 2 & 3 & 3 & 2.25 & - & DLB & $\mathrm{AD}$ & DLB \\
\hline 4 & Soccer & NA & High & 3 & 3 & 2 & 0 & 4 & CVD; ARTAG & Mixed $\mathrm{AD} / \mathrm{VaD}$ & CTE \\
\hline 5 & Soccer & $\mathrm{C}$ & Low & 3 & 3 & 2 & 3.5 & 3 & CVD; DLB; ARTAG & Mixed $\mathrm{AD} / \mathrm{VaD}$ & $\mathrm{AD}$ \\
\hline 6 & Soccer & Intact & No & 3 & 3 & 3 & 4 & - & ARTAG & 'early onset dementia' & $\mathrm{AD}$ \\
\hline 7 & Soccer & Intact & No & 0 & 0 & 0 & 0 & 4 & PSP & $\mathrm{AD}$ & PSP \\
\hline 8 & Rugby & $\mathrm{F}$ & High & 3 & 3 & 2 & 3 & 5 & ARTAG & $\mathrm{VaD}$ & CTE \\
\hline 9 & Rugby & $\mathrm{F}$ & Low & 3 & 3 & 3 & 4 & 4 & CVD; ARTAG & $\mathrm{AD}$ & $\mathrm{AD}$ \\
\hline 10 & Rugby & Intact & Low & 2 & 3 & 3 & 0.25 & 3 & CVD & $\mathrm{AD}$ & Mixed $\mathrm{AD} / \mathrm{VaD}$ \\
\hline 11 & Rugby & Intact & No & 0 & 0 & 0 & 0 & - & CBD & CTE & CBD \\
\hline
\end{tabular}

$A D$ Alzheimer's disease, $A D N C$ Alzheimer's disease neuropathologic changes, $A R T A G$ aging-related tau astrogliopathy, $C$ cavum, $C A A$ cerebral amyloid angiopathy [35]; $C B D$ corticobasal degeneration, $C P C$ clinicopathological correlation, $C T E-N C$ chronic traumatic encephalopathy neuropathologic change, $C V D$ chronic cerebrovascular disease, $D L B$ dementia with Lewy bodies, $F$ fenestrated, $N A$ not assessed, $N D$ neurodegenerative disease, $P A R T$ primary age-related tauopathy, $P S P$ progressive supranuclear palsy, $T D P-43$ abnormally phosphorylated TDP-43 pathology [16], $\mathrm{VaD}$ vascular dementia

[49], while in one case more localized (Thal phase 2) diffuse plaques were identified. In addition, cerebral amyloid angiopathy (CAA) was present in seven $\mathrm{A} \beta$ plaque-positive cases, typically of high severity by routinely employed histological assessment protocols [35].

\section{TDP-43}

Staining for phosphorylated TDP-43 revealed scattered neuronal cytoplasmic inclusions and neurites in seven brains, consistent with stage 3, 4 or 5 TDP-43 pathology [16]. This included Case 2, in which there was noted bilateral hippocampal sclerosis, supporting a pathological diagnosis of hippocampal sclerosis of aging [34]. The remaining cases were negative for TDP-43.

\section{Alpha-synuclein}

Alpha-synuclein-positive profiles were present in just two brains. In Case 3, abundant synuclein-immunoreactive neurites and neuronal cytoplasmic inclusions consistent with cortical Lewy bodies were present, supporting a neuropathologic diagnosis of diffuse neocortical pattern of Lewy body disease (Fig. 2d-f) [30, 39]. Case 5 exhibited classical Lewy bodies, with sparse synuclein-positive neurites and neurons in the cingulate gyrus, medial temporal lobe and amygdala supporting a diagnosis of limbic/transitional pattern Lewy body disease [30].

\section{Summary of neuropathologies}

Thus, multiple, mixed neurodegenerative pathologies were typical in these former soccer and rugby players with dementia. In some form, p-tau pathologies were identified in all cases. Thereafter, $\mathrm{A} \beta$ pathologies were present in $73 \%$, TDP-43 in 64\%, vascular pathology in 55\% and alpha-synuclein in $18 \%$. Deposition of p-tau in a pattern and distribution fulfilling preliminary neuropathological criteria for CTE was present in eight cases, all but one of which also showed mixed $p$-tau and $A \beta$ pathologies in keeping with Alzheimer's disease neuropathologic changes (ADNC), with seven cases in total showing 'high/intermediate' ADNC and a single case with 'low' ADNC [33]. In addition to plaque pathology, widespread CAA was present in seven brains, five of which showed features of macro- and/or micro-vascular ischemic pathology. A further two cases had evidence of ischemic pathology in the absence of CAA. All five cases with abnormalities in the septum pellucidum had co-existent CTE neuropathology.

\section{Integrated clinicopathological diagnosis}

Following neuropathological evaluation, verbal autopsy interviews with the donor's next of kin, supplemented by clinical case records' review, where available, provided comprehensive accounts of the clinical presentation and disease course (summarized in Table 3), which informed a final, integrated clinicopathological diagnosis of dementia subtype for each case. The most frequent integrated diagnosis was 
Alzheimer's disease (AD; four cases; one as mixed $\mathrm{AD}$ and vascular dementia), followed by three cases with dementia compatible with CTE (CTE-D). Of the remaining cases, these included one case each of vascular dementia, dementia with Lewy bodies, PSP and CBD. Thus, within this cohort, three broad diagnostic categories might be proposed, distinguished by the presence of CTE-NC and its association with dementia diagnosis. These consist of: (1) patients with dementia but no evidence of CTE-NC ( $n=3$; median age onset 50 years); (2) patients with an integrated diagnosis of CTE-D ( $n=3$; median 55 years); and (3) patients with CTE-NC as co-morbidity in context of an alternate integrated diagnosis $(n=5$; median 66 years; $p=0.024$; Kruskal-Wallis).

\section{Discussion}

Herein, we present observations on neurodegenerative pathologies and their relationship to the final integrated, clinicopathological dementia diagnosis in a consecutive series of 11 brain donations from former soccer and rugby union players with dementia. In addition to ubiquitous tau pathologies, our cases invariably showed mixed, often multiple, co-existent neurodegenerative pathologies, including frequent $\mathrm{A} \beta$ pathologies as amyloid plaques and cerebral amyloid angiopathy. Further, although the pathognomonic neuropathology of CTE described within preliminary consensus criteria was present in a high proportion of brains in this series, in only three would CTE be regarded as the primary integrated dementia diagnosis following comprehensive review of the clinical histories and neuropathologies. In the remaining five cases with CTE neuropathologic change (CTE-NC), this appeared as co-morbid pathology in the setting of an alternative integrated dementia diagnosis. Thus, in context of dementia arising in athletes exposed to repetitive mild traumatic brain injury, three distinct clinicopathological groupings are suggested by this series defined by the presence of CTE-NC and its relationship to the final integrated dementia diagnosis. These observations support the need for comprehensive neuropathological evaluation and reporting in individuals exposed to TBI, with distinction between the autopsy finding of CTE-NC and the putative clinical consequences of this pathology, including CTE dementia (CTE-D).

Notably, mixed neurodegenerative pathologies were present in nine of our series, even in relatively younger aged patients. Mixed pathologies increasingly are recognized in studies of neurodegenerative disease [18, 41, 44], with prevalence estimates ranging up to $80 \%$ of dementias at autopsy and directly correlated with age [41]. In contrast to our experience, previous series report mixed neurodegenerative pathologies in just $45 \%$ of former athletes [31]. In this respect, mixed pathologies appear more frequent in our patients than in similar studies in former athletes, perhaps reflecting an older patient cohort. Conceivably, such mixed neurodegenerative pathologies might contribute to clinically 'atypical' presentations, leading to challenges in establishing accurate clinical diagnoses. There is undoubtedly a need for continued autopsy brain examination in individuals surviving TBI, with accurate and comprehensive documentation of the full spectrum of pathologies encountered in each examination.

Reporting in CTE largely focuses on p-tau pathologies. Indeed, preliminary consensus criteria define pathognomonic CTE neuropathology solely by the pattern and distribution of p-tau [26]. While pathognomonic p-tau pathologies were common in our current series, $A \beta$ pathology as plaque and/or CAA was as frequent and present in all but one case with CTE-NC. In contrast, studies on former American footballers with CTE document $\mathrm{A} \beta$ pathology in just $61 \%$ [45]. However, incidence of dementia was reported higher in those older American footballers in which there were high burdens of CTE and A $\beta$. In this context, our data support these observations and might suggest that few postTBI dementias arise in context of a pure tauopathy (pure CTE-NC), the majority arising in context of mixed proteinopathies, including frequent $\mathrm{A} \beta$ pathologies. As such, dementia-associated neurodegenerative pathology in former athletes might be more reminiscent of AD.

Since the first description of CTE in a former American football player [36], there has been a proliferation in autopsy series reporting its presence in individuals surviving a spectrum of TBI exposures (for review, see Hay et al. [11]). Nevertheless, the published case experience in CTE remains low [43], with reports typically documenting CTE pathology, but including little interpretation of its clinical implications. By example, an autopsy series documenting 110 of 111 former National Football League (NFL) American footballers with CTE pathology offered no insights into final integrated clinicopathological diagnoses [31]. Similarly, no opinion on final, integrated clinicopathological diagnoses after neuropathological evaluation was provided in a study of six former soccer players with dementia, four of whom had CTE neuropathology [22]. We identified CTE-NC in eight of our 11 former athletes with dementia. However, in just three of these cases was the integrated, primary dementia diagnosis consistent with CTE dementia (CTE-D) after consideration of the comprehensive clinical information. As such, while CTE-NC is a common pathology in former contact sports athletes with dementia, CTE-D appears a less common primary dementia diagnosis. 

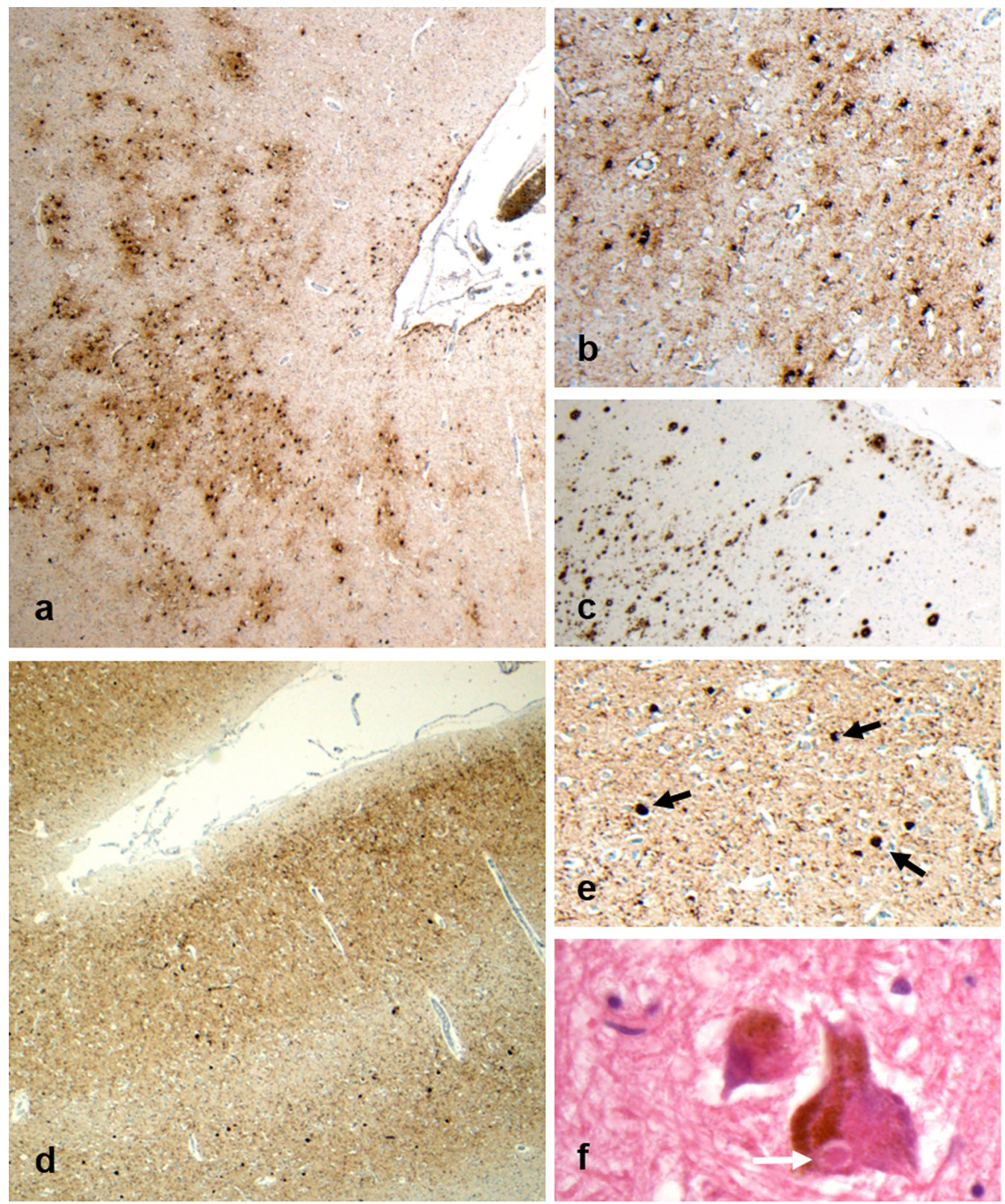
4Fig. 2 Representative images from Case 3, a former soccer player who first came to attention in his $60 \mathrm{~s}$ with mild cognitive impairment. There followed a steady progression in these symptoms, with exacerbations in the post-operative period, and with the development of visual hallucinations and motor signs leading to a clinical suspicion of Parkinson's disease, or related disorder. After an 8-year course, the patient died in his $70 \mathrm{~s}$. Staining for $\mathrm{p}$-tau reveals patches of neuronal and astroglial immunoreactivity at the depths of cortical sulci (a), which on higher power are clustered around small cortical vessels (b; PHF1). Frequent A $\beta$ plaques are also present (c; 6f3d). In addition, staining for alpha-synuclein reveals extensive cortical staining (d), with numerous cortical Lewy bodies present (e; black arrows). Sections of the midbrain also reveal scattered classical Lewy bodies (f; white arrow) within remaining pigmented neurons of the substantia nigra. Given the stereotypical clinical presentation and associated neuropathological features, an integrated diagnosis of dementia with Lewy bodies was made, with co-morbid CTE-NC noted

In this series of former soccer and rugby players with dementia, CTE-NC was present in $73 \%$ of cases. Adding this experience to observations in previous, isolated case reports in rugby and soccer, where sought, autopsy confirmed CTE pathology is reported in $75 \%$ of former athletes from these sports: 13/18 (seven from this study) soccer [1, 6, 9, 10, $22,25]$ and $5 / 6$ (four from this study) rugby [25, 45]. Elsewhere, CTE neuropathology has been reported ranging from $50 \%$ (7/14) of former boxers [7] to 99\% (110/111) of former NFL footballers examined [31]. Although such studies are subject to numerous biases and limitations, rendering them uninformative regarding true disease prevalence, the apparent variation in reported prevalence of CTE neuropathology between these autopsy series might suggest between sports' differences in risk and/or variability in methodologies of specimen accrual and neuropathology assessment between laboratories.

In summary, our observations suggest that while CTE-NC may be a common pathology in former soccer and rugby players with dementia, CTE dementia appears a less common primary dementia diagnosis. Current criteria for $A D$ distinguish neurodegenerative pathologies, defined as $A D$ neuropathologic changes (ADNC) [13,33], from the clinical syndrome of dementia of Alzheimer's disease type [14]. Reflecting this approach, we propose that there should be a similar distinction between CTE neuropathologic change and the clinical consequences of this pathology, including CTE dementia. As experience grows, CTE-NC conceivably might echo experience in $\mathrm{AD}$, where the neuropathologic change can represent incidental pathology without clinical effect, or the major pathology driving clinical dementia phenotypes, or a co-morbid pathology within mixed neurodegenerative disease. In other words, in the absence of clinical correlation, CTE neuropathologic change should perhaps be regarded as a pattern of pathology, rather than diagnostic of a specific disease.

Table 3 Major clinical symptoms reported

\begin{tabular}{|c|c|c|c|c|}
\hline Case no & $\begin{array}{l}\text { Age } \\
\text { symptom } \\
\text { onset }\end{array}$ & Age at death & $\begin{array}{l}\text { Disease } \\
\text { duration } \\
\text { (years) }\end{array}$ & Major symptoms at presentation and over disease course \\
\hline 1 & $50 \mathrm{~s}$ & $50 \mathrm{~s}$ & 5 & $\begin{array}{l}\text { Behavioral change and personality change (impulsivity) at onset; progression to cognitive } \\
\text { impairment (memory) late in disease }\end{array}$ \\
\hline 2 & $60 \mathrm{~s}$ & $60 \mathrm{~s}$ & 7 & $\begin{array}{l}\text { Mild, but evolving cognitive impairment (memory) at onset; acute stroke with cognitive } \\
\text { deterioration later in disease and stepwise deterioration thereafter }\end{array}$ \\
\hline 3 & $60 \mathrm{~s}$ & $70 \mathrm{~s}$ & 8 & $\begin{array}{l}\text { Cognitive impairment (problem solving) at onset; acute deterioration post-operatively; Par- } \\
\text { kinsonism and visual hallucinations late in course }\end{array}$ \\
\hline 4 & $60 \mathrm{~s}$ & $70 \mathrm{~s}$ & 10 & $\begin{array}{l}\text { Behavioral change with aggression and cognitive impairment (memory) at onset; Reduced } \\
\text { mobility late in disease }\end{array}$ \\
\hline 5 & $60 \mathrm{~s}$ & $80 \mathrm{~s}$ & 16 & $\begin{array}{l}\text { Loss of confidence associated with cognitive impairment (spatial awareness) at onset; pro- } \\
\text { gressing memory impairment; sleep and behavioral disturbance late in course }\end{array}$ \\
\hline 6 & $50 \mathrm{~s}$ & $50 \mathrm{~s}$ & 6 & $\begin{array}{l}\text { Cognitive impairment (memory; conceptual) at onset; behavioral change later as mild disin- } \\
\text { hibition }\end{array}$ \\
\hline 7 & $50 \mathrm{~s}$ & $60 \mathrm{~s}$ & 13 & Behavioral change at onset; progressing to memory decline and motor symptoms \\
\hline 8 & $50 \mathrm{~s}$ & $70 \mathrm{~s}$ & 18 & $\begin{array}{l}\text { Behavioral and personality change (short tempered; irritable; loss of confidence); memory } \\
\text { impairment late in disease }\end{array}$ \\
\hline 9 & $60 \mathrm{~s}$ & $70 \mathrm{~s}$ & 16 & $\begin{array}{l}\text { Cognitive impairment (memory) at onset; gradual decline with evolving cognitive symptoms } \\
\text { thereafter; not behavioral symptoms }\end{array}$ \\
\hline 10 & $70 \mathrm{~s}$ & $70 \mathrm{~s}$ & 4 & Cognitive impairment post diagnosed lacunar infarct at onset; decline thereafter \\
\hline 11 & $50 \mathrm{~s}$ & $50 \mathrm{~s}$ & 7 & $\begin{array}{l}\text { Behavioral change (becoming introverted) and mild cognitive (memory) symptoms at onset; } \\
\text { motor symptoms (shuffling gait) and speech loss with progression }\end{array}$ \\
\hline
\end{tabular}


Acknowledgements This work is supported by funding from: the National Institute of Neurological Disorders and Stroke of the National Institutes of Health under award numbers R01NS092398, R01NS094003 and R01NS038104; The Football Association and Professional Footballers Association (WS); and an NHS Research Scotland Career Researcher Fellowship (WS).

Author contributions WS and KK reviewed the pathology in each case and generated the original observations and dataset; WS provided a first draft of the manuscript and collated comments into a final submission draft. All other authors contributed to discussions on data analysis and interpretation and provided edits and comments on manuscript drafts.

\section{Compliance with ethical standards}

Conflict of interest The authors declare no competing interests.

Open Access This article is distributed under the terms of the Creative Commons Attribution 4.0 International License (http://creativeco mmons.org/licenses/by/4.0/), which permits unrestricted use, distribution, and reproduction in any medium, provided you give appropriate credit to the original author(s) and the source, provide a link to the Creative Commons license, and indicate if changes were made.

\section{References}

1. Bieniek KF, Ross OA, Cormier KA, Walton RL, Soto-Ortolaza A, Johnston AE et al (2015) Chronic traumatic encephalopathy pathology in a neurodegenerative disorders brain bank. Acta Neuropathol 130:877-889

2. Braak H, Braak E (1995) Staging of Alzheimer's disease-related neurofibrillary changes. Neurobiol Aging 16:271-278

3. Brandenburg W, Hallervorden J (1954) Dementia pugilistica with anatomical findings [German]. Virchows Arch Pathol Anat Physiol Klin Med 325:680-709

4. Corsellis JA, Bruton CJ, Freeman- Browne D (1973) The aftermath of boxing. Psychol Med 3:270-303

5. Dickson DW, Bergeron C, Chin SS, Duyckaerts C, Horoupian D, Ikeda K et al (2002) Office of rare diseases neuropathologic criteria for corticobasal degeneration. J Neuropathol Exp Neurol 61:935-946

6. Geddes JF, Vowles GH, Nicoll JA, Revesz T (1999) Neuronal cytoskeletal changes are an early consequence of repetitive head injury. Acta Neuropathol 98:171-178

7. Goldfinger MH, Ling H, Tilley BS, Liu AKL, Davey K, Holton JL, Revesz T, Gentleman SM (2018) The aftermath of boxing revisited: identifying chronic traumatic encephalopathy pathology in the original Corsellis boxer series. Acta Neuropahtolgica 136:973-974

8. Goldstein LE, Fisher AM, Tagge CA, Zhang XL, Velisek L, Sullivan JA et al (2012) Chronic traumatic encephalopathy in blastexposed military veterans and a blast neurotrauma mouse model. Sci Transl Med 4:134ra160

9. Grinberg LT, Anghinah R, Nascimento CF, Amaro E, Leite RP, Martin MGM et al (2016) Chronic traumatic encephalopathy presenting as Alzheimer's disease in a retired soccer player. J Alzheimers Dis 54:169-174

10. Hales C, Neill S, Gearing M, Cooper D, Glass J, Lah J (2014) Late-stage CTE pathology in a retired soccer player with dementia. Neurology 83:2307-2309
11. Hay J, Johnson VE, Smith DH, Stewart W (2016) Chronic traumatic encephalopathy: the neuropathological legacy of traumatic brain injury. Ann Rev Pathol 11:21-45

12. Hof PR, Knabe R, Bovier P, Bouras C (1991) Neuropathological observations in a case of autism presenting with self-injury behavior. Acta Neuropathol 82:321-326

13. Hyman BT, Phelps CH, Beach TG, Bigio EH, Cairns NJ, Carrillo MC et al (2012) National Institute on Aging-Alzheimer's Association guidelines for the neuropathologic assessment of Alzheimer's disease. Alzheimers Dement 8:1-13

14. Jack CR Jr, Albert MS, Knopman DS, McKhann GM, Sperling RA, Carrillo MC, Thies B, Phelps CH (2011) Introduction to the recommendations from the National Institute on Aging-Alzheimer's Association workgroups on diagnostic guidelines for Alzheimer's disease. Alzheimers Dement 7:257-262

15. Johnson VE, Stewart W, Smith DH (2012) Widespread tau and amyloid-beta pathology many years after a single traumatic brain injury in humans. Brain Pathol 22:142-149

16. Josephs KA, Murray ME, Whitwell JL, Tosakulwong N, Weigand SD, Petrucelli L, Liesinger AM, Petersen RC, Parisi JE, Dickson DW (2016) Updated TDP-43 in Alzheimer's disease staging scheme. Acta Neuropathol 131:571-585

17. Kovacs GG (2015) Neuropathology of tauopathies: principles and practice. Neuropathol Appl Neurobiol 41:3-23

18. Kovacs GG, Alafuzoff I, Al-Sarraj S, Arzberger T, Bogdanovic N, Capellari S et al (2008) Mixed brain pathologies in dementia: the BrainNet Europe Consortium experience. Dement Geriatr Cogn Disord 26:343-350

19. Kovacs GG, Ferrer I, Grinberg LT, Alafuzoff I, Attems J, Budka $\mathrm{H}$ et al (2016) Aging-related tau astrogliopathy (ARTAG): harmonized evaluation strategy. Acta Neuropathol 131:87-102

20. Kovacs GG, Xie SX, Lee EB, Robinson JL, Casell C, Irwin DJ et al (2017) Multisite assessment of aging-related tau astrogliopathy (ARTAG). J Neuropathol Exp Neurol 76:605-619

21. Lee EB (2018) Integrated neurodegenerative disease autopsy diagnosis. Acta Neuropathol 135:643-646

22. Ling H, Morris HR, Neal JW, Lees AJ, Hardy J, Holton JL, Revesz T, Williams DDR (2017) Mixed pathologies including chronic traumatic encephalopathy account for dementia in retired association football (soccer) players. Acta Neuropathol 133:337-352

23. Litvan I, Haum JJ, Bartko JJ, Lantos PL, Daniel SE, Horoupian DS et al (1996) Validity and reliability of the preliminary NINDS neuropathologic criteria for progressive supra-nuclear palsy and related disorders. J Neuropathol Exp Neurol 55:97-105

24. Martland H (1928) Punch drunk. JAMA 91:1103-1107

25. McKee AC, Daneshvar DH, Alvarez VE, Stein TD (2014) The neuropathology of sport. Acta Neuropathol 127:29-51

26. McKee AC, Cairns NJ, Dickson DW, Folkerth RD, Keene CD, Litvan I et al (2016) The first NINDS/NIBIB consensus meeting to define neuropathological criteria for the diagnosis of chronic traumatic encephalopathy. Acta Neuropathol 131:75-86

27. McKee AC, Cantu RC, Nowinski CJ, Hedley-Whyte ET, Gavett BE, Budson AE et al (2009) Chronic traumatic encephalopathy in athletes: progressive tauopathy after repetitive head injury. J Neuropathol Exp Neurol 68:709-735

28. McKee AC, Stein TD, Nowinski CJ, Stein TD, Alvarez VE, Daneshvar DH et al (2012) The spectrum of disease in chronic traumatic encephalopathy. Brain 136:43-64

29. McKee AC, Stein TD, Nowinski CJ, Stern RA, Deneshvar DH, Alvarez VE et al (2012) The spectrum of disease in chronic traumatic encephalopathy. Brain 136:43-64

30. McKeith IG, Boeve BF, Dickson DW, Halliday G, Taylor JP, Weintraub D et al (2017) Diagnosis and management of dementia with Lewy bodies: fourth consensus report of the DLB consortium. Neurology 89:88-100 
31. Mez J, Daneshvar DH, Kiernan PT, Abdolmohammadi B, Alvarez VE, Huber BR et al (2017) Clinicopathological evaluation of chronic traumatic encephalopathy in players of American football. JAMA 318:360-370

32. Mirra SS, Heyman A, McKeel D, Sumi SM, Crain BJ, Brownlee LM et al (1991) The consortium to establish a registry for Alzheimer's disease (CERAD). Part II. Standardization of the neuropathologic assessment of Alzheimer's disease. Neurology 41:479-486

33. Montine TJ, Phelps CH, Beach TG, Bogio EH, Cairns NJ, Dickson SW et al (2012) National Institute on Aging and Alzheimer's guidelines for the neuropathologic assessment of Alzheimer's disease: a practical approach. Acta Neuropathol 123:1-11

34. Nag S, Yu L, Capuano AW, Wilson RS, Leurgans SE, Bennett Da, Schneider JA (2015) Hippocampal sclerosis and TDP-43 pathology in aging and Alzheimer's disease. Ann Neurol 77:942-952

35. Olichney JM, Hansen LA, Hofstetter CR, Grundman M, Katzman R, Thal LJ (1995) Cerebral infarction in Alzheimer's disease is associated with severe amyloid angiopathy and hypertension. Arch Neurol 52:702-708

36. Omalu BI, DeKosky ST, Minster RL, Kamboh MI, Hamilton RL, Wecht CH (2005) Chronic traumatic encephalopathy in a National Football League player. Neurosurgery 57:128-134

37. Omalu BI, Fitzsimmons RP, Hammers J, Bailes J (2010) Chronic traumatic encephalopathy in a professional American wrestler. J Forensic Nurs 6:130-136

38. Omalu B, Hammers JL, Bailes J, Hamilton RL, Kamboch MI, Webster G, Fitzsimmons RP (2011) Chronic traumatic encephalopathy in an Iraqi war veteran with posttraumatic stress disorder who committed suicide. Neurosurg Focus 31:E3

39. Outeiro TF, Koss DJ, Erskine D, Walker L, Kurzawa-Akanabi M, Burn D et al (2019) Dementia with Lewy bodies: an update and outlook. Mol Neurodegener 14:5. https://doi.org/10.1186/s1302 4-019-0306-8

40. Roberts GW, Allsop D, Bruton C (1990) The occult aftermath of boxing. J Neurol Neurosurg Psychiatry 53:373-378

41. Robinson J, Lee EB, Xie SX, Rennert L, Suh E, Bredenberg C et al (2018) Neurodegenerative disease concomitant proteinopathies are prevalent, age-related and APOE4- associated. Brain 141:2181-2193
42. Smith DH, Johnson VE, Stewart W (2013) Chronic neuropathologies of single and repetitive TBI: substrates of dementia? Nat Rev Neurol 9:211-221

43. Smith DH, Johnson VE, Trojanowski JQ, Stewart W (2019) Chronic traumatic encephalopathy-confusion and controversies. Nat Rev Neurol 15:179-183

44. Spires-Jones TL, Attems J, Thal DR (2017) Interactions of neuropathological proteins in neurodegenerative diseases. Acta Neuropathol 134:187-205

45. Stein TD, Montenigro PH, Alvarez VE, Xia W, Crary JF, Tripodis Y et al (2015) Beta-amyloid deposition in chronic traumatic encephalopathy. Acta Neuropathol 130:21-34

46. Stern RA, Daneshvar DH, Baugh CM, Seichepine DR, Montenigro PH, Riley DO et al (2013) Clinical presentation of chronic traumatic encephalopathy. Neurology 2:1122-1129

47. Stewart W, McNamara PH, Lawlor B, Hutchinson S, Farrell M (2016) Chronic traumatic encephalopathy: a potential late and under recognized consequence of rugby union? QJM 109:11-15

48. Stewart W, Allinson K, Al-Sarraj S, Bachmeier C, Barlow K, Belli A (2019) Primum non nocere: a call for balance when reporting on CTE. Lancet Neurol 18:231-233

49. Thal DR, Rub U, Orantes M, Braak H (2002) Phases of $\mathrm{A} \beta$-deposition in the human brain and its relevance for the development of AD. Neurology 58:1791-1800

50. Wilson L, Stewart W, Dams-OConnor K, Diaz-Arrastia R, Horton L, Menon DK, Polinder S (2017) The chronic and evolving neurological consequences of traumatic brain injury. Lancet Neurol 16:813-825

51. Zanier ER, Bertani I, Sammali E, Pischiutta F, Chiaravalloti MA, Vegliane $G$ et al (2018) Induction of a transmissible tau pathology by traumatic brain injury. Brain 141:2685-2699

Publisher's Note Springer Nature remains neutral with regard to jurisdictional claims in published maps and institutional affiliations. 